\title{
A Theoretical Approach for Understanding the Haze Phenomenon in Bottled White Wines at Molecular Level
}

\author{
L.N. Toledo ${ }^{1 *}$, F.N. Salazar ${ }^{1,2}$, A.J.A. Aquino ${ }^{3}$ \\ (1) Escuela de Ingeniería de Alimentos, Avenida Waddington \#716, Playa Ancha, Pontificia Universidad Católica de Valparaíso, \\ Valparaíso, Chile \\ (2) Laboratorio de Fermentaciones industriales (IFELab), Avenida Waddington \#716, Playa Ancha, Pontificia Universidad \\ Católica de Valparaíso, Valparaíso, Chile \\ (3) Department of Chemistry and Biochemistry, Texas Tech University TX 79409-1061, USA
}

Submitted for publication: August 2016

Accepted for publication: December 2016

Key words: Energy of binding, haze, thaumatin-like proteins, Vitis vinifera, bottled wine

\begin{abstract}
The stabilisation of the proteinaceous material in the wine matrix represents one of the big challenges for the production of quality white wines, but the characterisation of the mechanism that governs the interactions between its components is still a very challenging goal. The aim of this study was to provide new information for developing new technologies in the stabilisation of bottled white wines using a novel theoretical approach. This method combines electronic structure calculations for the determination of the a stable conformation of three ligands that may interact with one of the proteins responsible for the haze in wines, the thaumatin-like protein (TLP), with the search for the mode of binding between this protein and its ligands through docking calculations. The result shows that sites that exposed positive residues to the surface of the protein are the sites favoured for the caffeic acid (CA) binding. Additionally, it was observed that the ligand with the lowest binding energy $(-7.38 \mathrm{kcal} / \mathrm{mol})$ was the quercetin $(Q)$. The presence of a $\pi-\pi$ stacking interaction with the residue F118 is confirmed in a family of TLP-Q complexes, and it is proposed that the mechanism of haze formation in white wines during bottle storage seems to be related to the interaction of polyphenolic molecules with some residues of this big cavity; these residues or sites of interaction can be considered as future targets in the control of the haze phenomena and in the research on alternatives to the fining treatment in the wine industry.
\end{abstract}

\section{INTRODUCTION}

The genesis of haze formation in white wine has been described as a multi-factorial problem, in which proteinaceous components, nonproteinaceous components and media conditions, such as metal ions, $\mathrm{pH}$, ionic strength, polysaccharides and phenolic compounds, seem to play a role (Waters et al., 2005; Pocock et al., 2007; Marangon et al., 2011a). Experimental trials have been focused on determining the mechanism behind the haze formation in the bottle once the product has passed the winemaking process. Although this haze does not present a health risk (Marangon et al., 2011) or affect the organoleptic quality of the wine (Peng et al., 1997), its presence is perceived negatively as a loss of quality by the consumers.

Thaumatin-like proteins (TLP) and chitinases are the major soluble proteins of grape berries (Tattersall et al., 1997) and have been deemed responsible for haze formation in white wines (Dawes et al., 1994). Chitinases are a family of proteins with a low molecular weight and are sensitive to changes in temperature (Falconer et al., 2010) and
pH (Dufrechou et al., 2013). On the other hand, the TLP family is characterised principally by its thermostability and by showing no significant conformational changes or aggregation when exposed to changes in the $\mathrm{pH}$ (Dufrechou et al., 2013). This unequal behaviour seems to be related to the differences in the secondary structure of both families, which has been described as globular and elliptical for TLP and chitinases respectively (Tattersall et al., 2001; Dufrechou et al., 2013).

Experimental studies have provided valuable information for identifying the agents that could be present and be responsible for triggering the haze formation: phenolic compounds (Heatherbell, 1976), polysaccharides (Pellerin et al., 1994), metals as copper (Besse et al., 2000), and other inorganic molecules (Pocock et al., 2007).

At the industrial level, the use of benthonic earths during the operation of fining is the most common and effective treatment to remove the proteinaceous material from the wines. However, it is claimed that its use adversely affects 
the quality of the treated wine, because the bentonite is a non-selective entrainer agent, which under certain conditions can remove colour, flavour and texture compounds from the matrix of the product (Høj et al., 2000). It has been estimated that, for the worldwide production of wine, the cost of the bentonite fining treatment is in the order of US\$300 million to US\$500 million per year (Høj et al., 2000), and that around $3 \%$ to $10 \%$ of the wine volume is lost by the bentonite lees in the fining treatment (Tattersall et al., 2001).

Studying the wine matrix to advance the comprehensive understanding of the haze phenomenon poses a huge challenge for researchers, considering all the potential interactions that can occur between the diversity of macromolecules that coexist in the matrix of this product. The use of model wines has been tested to simplify this aspect. In this kind of matrix only target molecules, such as ethanol, proteins and some non-proteinaceous material, are considered for its formulation (Yokotsuka et al., 1991; Picinelli et al., 1994). Even though valuable information has been obtained, in some cases the results are non-reproducible under real conditions in commercial wines (Waters et al., 1995).

This work uses an innovative approach to study the genesis of the mechanism of haze formation. As starting point, the recognition between the TLP and three ligands at molecular level is considered by means of molecular modelling. This would help to predict trends that are not accessible by traditional experimental methods in one of the proteins that seems to trigger haze formation in the long term. It is expected that this information can be scrutinised experimentally in the future as an alternative and effective method to bentonite fining treatment. In this sense, this study aims to contribute to the understanding of the mechanism that governs the haze phenomenon in bottled wines (long time) through bioinformatic tools, taking into account, at the molecular level, the binding mechanism of the complexes formed between TLP and three potential ligands: caffeic acid, quercetin and sulphate.

To our knowledge, this strategy has not been used before in this field and presents a complete innovation in the understanding of the haze phenomenon in bottled white wines.

\section{MATERIALS AND METHODS}

The methodology used in this research works with the three-dimensional published structures of the protein and the ligands and predicts its most favoured complex under energetic criteria, allowing for a detailed description, at the molecular level, of the site(s) of binding, the kind of interactions, and an energetic rank of the complexes grouped as conformational families (clusters).

The methods that are used in this research have been used intensively in drug design (Hellberg et al., 1987; Wishart et al., 2006; Matta \& Boyd, 2007; Yuan et al., 2013; Distinto et al., 2015; Kristensen et al., 2015; Lin, 2016; Wang et al., 2016; Xiao et al., 2016), and it is hoped that their use in this field complements the experimental information from a new approach, favouring the holistic comprehension of the phenomenon.

\section{Obtaining the minimised structures}

The protein structure used in this study corresponds to the $\mathrm{X}$-ray structure of the TLP from Vitis vinifera white grapes at a resolution of $1.20 \AA$ (Marangon et al., 2014), taken from the Protein Data Bank (PDB) (Bernstein et al., 1977). It is important to note that TLP is the only Vitis vinifera species for which the X-ray crystallographic structure is available in the PDB. The hydrogen atoms of each residue in the structure were assigned according to the semi-empirical description included in the force field of the program CHARMM (Brooks et al., 1983), using the facility Hbuild. For the titratable residues of the structure, a Poisson-Boltzmann electrostatics calculation was considered, following the work published by Dolinsky et al. (2004). The evaluation of the protonation state of the protein at $\mathrm{pH} 2.5$ and $\mathrm{pH} 4.0$ was performed by taking into account the results of a previous experimental study (Dufrechou et al., 2013) and its potential effect on the mode of binding of the ligands. The structure was minimised to prevent steric clashes between the added atoms and those determined by X-ray, using 100 steps of the steepest descent algorithm and 100 steps of the adapted-basis Newton-Raphson method, also available in the program cited previously (CHARMM).

For a precise description of the most stable conformation of the ligands (sulphate, caffeic acid and quercetin), geometry optimisation calculations were performed in relation to the scaled opposite-spin second-order Moller-Plesset perturbation theory (SOS/MP2) level (Jung et al., 2004) and the aug-cc-pVDZ basis set (Kendall et al., 1992). To confirm that the selected geometry corresponds to a minimum in the potential energy curve, the harmonic frequency calculations for the optimised structure of each ligand were carried out. Atomic charges were also computed through natural population analysis (NPA) (Reed et al., 1985) as available in the Turbomole program suite (Ahlrichs et al., 1989). This computational code was used for all geometry and frequency calculations of the ligands.

The initial superimposition of the structures in the same virtual space was done using the tool Match from the Chimera software (Pettersen et al., 2004). At this stage, the coupling calculations (called docking calculations) between each ligand and the TLP structure were performed considering a semi-rigid approach as described in full below.

\section{Docking calculations}

The computational docking calculations typically performed a search based on the framework of the molecular mechanics methods, in which the atoms in molecules are treated as rubber balls of different sizes (atom types) joined together by springs of varying lengths (bonds). This enables the calculation of the total energy of the system in terms of deviation from reference unstrained bond lengths, angles and torsions plus the non-bonded interactions, giving rise to the force constant for the molecules, which under empirically derived fit is known as the force field (Höltje et al., 2008). The calculations explore a wide region of the selected space on the protein to predict the more favoured mode of binding and point of anchor, with no a priori knowledge of these binding sites on the protein (Huey et al., 2007). In this work, the energetic space of the search over the protein structure 
(called the grid) was established through $70 \times 104 \times 106$ points by axis, which cover all the molecules of TLP, with a spacing of $0.375 \AA$ between each point to calculate the grid maps, which define the energetic space of search over the total structure in the macromolecule. With the information from the grid maps it was possible to generate new individuals based on the structural information of the molecules (torsion points), denominated a Generation, from which were selected the more favoured individual through a local search using the Lamarckian Genetic Algorithm (Morris et al., 1998). The categorisation of the individuals in energetic terms is done afterwards, through the scoring function that considers the steric, hydrogen bonding, hydrophobic and torsional entropy terms present in the force field of version 4.0 of the software Autodock (Huey et al., 2007). As a result of these calculations, 300 models of the complexes for each ligand were obtained (900 models in total). The results were ranked according to the energetic criteria and were named $\mathrm{C}$ families, those complexes between the TLP and caffeic acid; $\mathrm{Q}$ families, the complexes between the TLP and quercetin; and $\mathrm{S}$ families, for the complexes with the sulphate molecule.

\section{RESULTS}

Coupling the structures to the $\mathrm{pH}$ conditions in white wines

The initial X-ray structures from the PDB for the TLP and ligands were downloaded and adapted by different protocols, as explained in the previous section. Dufrechou et al. (2013) mentioned that the X-ray structure of the TLP has a compact secondary structure with a huge exposure surface and a big cavity, described by the residues exposed to the surface as R67, Y98, F118, N179 and K204, and at the bottom the residues D120, N122 and Y200, as shown in Fig. 1.

With the aim to reproduce in detail the structure of titratable residues of the TLP and to characterise as closely as possible the behaviour of the proteins in this static approach, we used the Propka program (Dolinsky et al., 2004; Li et al., 2005), which predicts the $\mathrm{pKa}$ values of ionisable groups in the protein based on its 3D structure.

Bearing in mind previous research that evaluated the correlation between the behaviour at two different conditions of pH (Dufrechou et al., 2013), the values used for the theoretical evaluation were the same used in that research, at $\mathrm{pH} 2.5$ and 4.0 respectively.

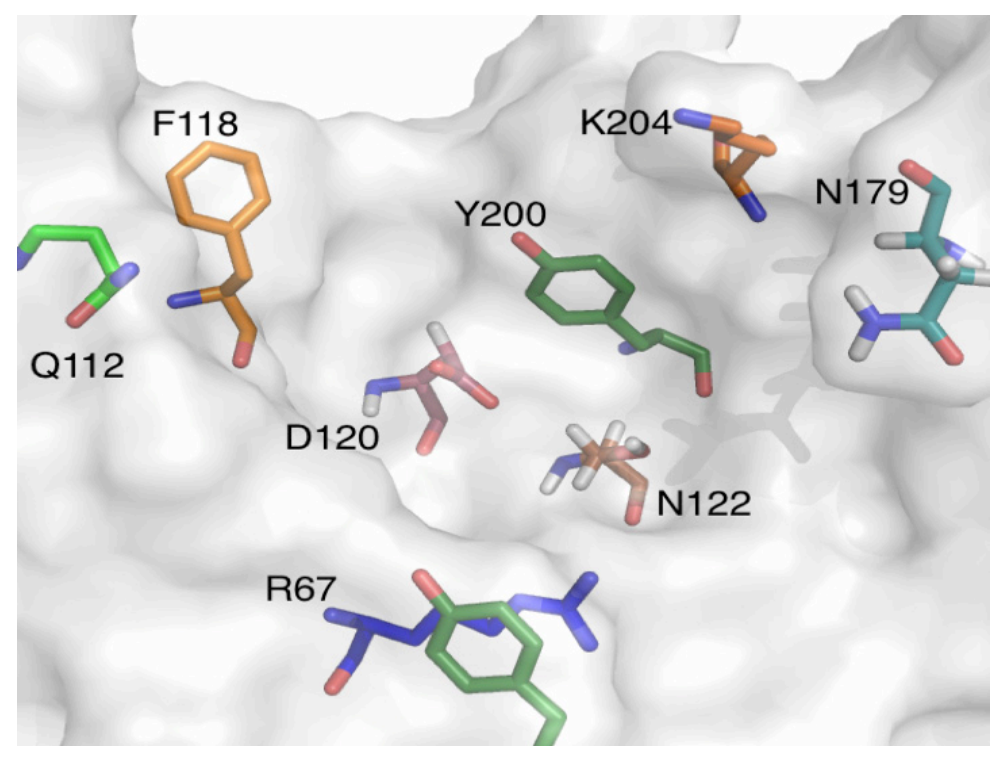

FIGURE 1

Representation in sticks of the amino acids that modulate the space of the biggest hydrophobic cavity on the TLP (represented as surface).
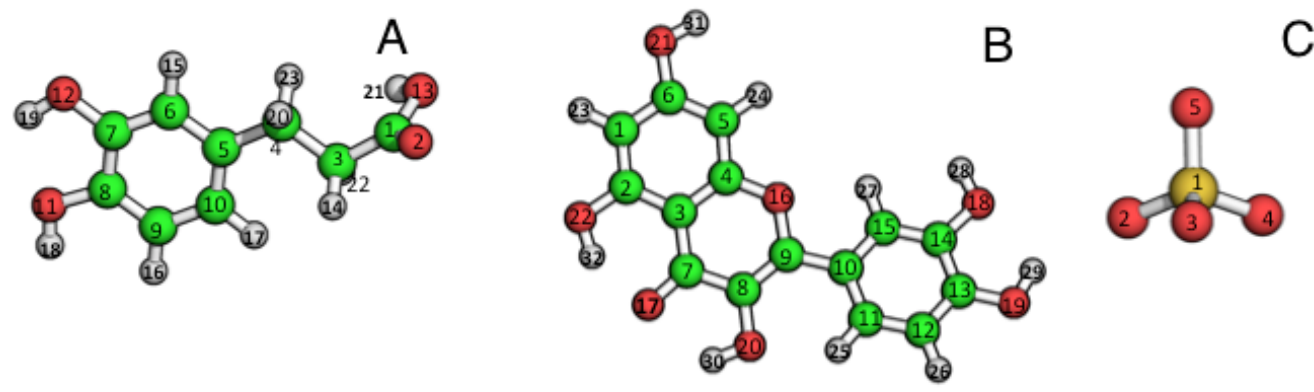

FIGURE 2

Optimised structures obtained through electronical calculations for the a) caffeic acid, b) quercetin and c) sulphate. 
There were no significant differences in the dissociation constant of the titratable side chain residues between $\mathrm{pH} 2.5$ and $\mathrm{pH} 4.0$, presumably because most of these residues are immersed inside the globular structure, in which $85 \%$ of residues have more than $50 \%$ of their atoms buried. These results are in agreement with the experimental trials of Dufrechou et al. (2013), in which no structural differences were observed at $\mathrm{pH} 2.5$ versus 4.0, measured by Small Angle $\mathrm{X}$-ray Scattering (SAXS) and fluorescence spectroscopy at the same temperature. Finally, considering the $\mathrm{pH}$ of the environmental in the wine matrix, the state of protonation of the titratable residues on the exposed protein surface were defined as protonated.

\section{Protein and ligand minimisation}

Although the minimisation of the structures allows reaching improved results on the structures, it is important to mention that, in this study, only the hydrogen atoms in the protein were minimised and for this reason it is not possible to infer information about the dynamic behaviour of the secondary structure over time, and its interaction with each one of the ligands, principally due to the lack of parameters for them in the force field of CHARMM.

The optimised geometry of the ligands by means of the SOSMP2/aug-cc-pVDZ is presented in Fig. 2. The frequency calculations of these converged structures did not show any imaginary values. This means that they correspond to an energy minimum in a potential curve and were used to calculate the atomic charge of the non-bonded interactions in the force field of the docking program.

As seen in Fig. 2, the molecule of sulphate has a tetrahedral configuration in its global minimum, and for the caffeic acid and the quercetin molecule the phenolic ring(s) determine their almost planar structure. The calculated NPA charges determined for each atom of the ligand by the electronic calculations (Table 1) were considered in the respective files that specify the charges of the ligands for the docking calculations.

\section{Docking calculations}

The results of the docking calculations were analysed by first considering the clustering information present in the histograms, which indicate the dispersion of the results based on a grouping criterion. This criterion defines the ligand that presents the lowest binding energy (without the protein) as the anchor, and then the next model in energy terms is compared with it through the RMSD value, under a threshold of $2.0 \AA$, as mentioned before. When all the models that meet the conformational requirement have been grouped and ranked according to their energy of binding, a new anchor is generated and the procedure continues until the last ligand (with the biggest energy of binding) has been analysed. In this sense, the three ligands evaluated have a very low dispersion, with 10 clusters for the caffeic acid, 17 for the quercetin and three for the sulphate molecule (see Fig. 3). This fact indicates that there are only a few zones accessible for the interaction with these ligands in the protein. Additionally, it is important to mention that three different clusters of CA are binding to the same zones of the protein. The only difference between these families is the value of the RMSD beyond $2.0 \AA$, or the orientation adopted by the ligand, indicating that this region of the surface of the TLP is highly favoured by this ligand in energetic terms. In the case of the quercetin, 14 of the 17 families (287 models out of 300) are on the same site of the protein, while for the caffeic acid there are two more favoured zones of contact, and one for the sulphate (see Fig. 4).

It is very interesting to note that the values of the energy binding for quercetin are the lowest compared with the energy values for the caffeic acid and sulphate molecule and, from an energetic point of view, seem to be the most stable under the conditions studied. In Table 2 the lowest binding energy of the complex, ranked 1 for cluster 1 , and the average energy of binding for this whole first family, are presented for the three ligands.

\section{TABLE 1}

Natural atomic charges (e) per atom determined by geometry optimisation calculations in the structures of the ligands.

\begin{tabular}{|c|c|c|c|}
\hline Atom number & Caffeic acid & Quercetin & Sulphate \\
\hline 1 & 0.84364 & 0.33966 & 2.46321 \\
\hline 2 & -0.59381 & 0.40566 & -0.61384 \\
\hline 3 & -0.54033 & -0.26453 & -0.61461 \\
\hline 4 & -0.44258 & 0.39609 & -0.61754 \\
\hline 5 & -0.05384 & -0.37847 & -0.61721 \\
\hline 6 & -0.25843 & 0.37222 & \\
\hline 7 & 0.29472 & 0.46498 & \\
\hline 8 & 0.25412 & 0.21031 & \\
\hline 9 & -0.28285 & 0.32709 & \\
\hline 10 & -0.24960 & -0.12374 & \\
\hline 11 & -0.73396 & -0.19458 & \\
\hline 12 & -0.70705 & -0.26018 & \\
\hline 13 & -0.71978 & 0.29994 & \\
\hline 14 & 0.26002 & 0.26123 & \\
\hline 15 & 0.24340 & -0.26399 & \\
\hline 16 & 0.23062 & -0.49316 & \\
\hline 17 & 0.23195 & -0.69055 & \\
\hline 18 & 0.50282 & -0.73239 & \\
\hline 19 & 0.50858 & -0.69993 & \\
\hline 20 & 0.23451 & -0.71051 & \\
\hline 21 & 0.49311 & -0.69226 & \\
\hline 22 & 0.23087 & -0.70260 & \\
\hline 23 & 0.25387 & 0.25954 & \\
\hline 24 & & 0.24036 & \\
\hline 25 & & 0.25995 & \\
\hline 26 & & 0.24899 & \\
\hline 27 & & 0.24201 & \\
\hline 28 & & 0.50368 & \\
\hline 29 & & 0.50982 & \\
\hline 30 & & 0.52106 & \\
\hline 31 & & 0.49340 & \\
\hline 32 & & 0.53019 & \\
\hline
\end{tabular}

Atoms numbered according to the numbers presented in Fig. 2. 

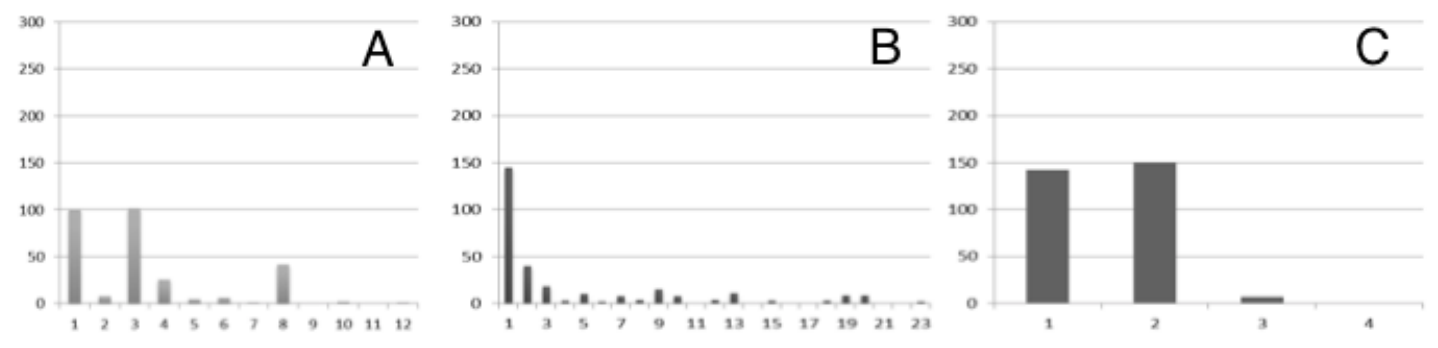

FIGURE 3

Number of clusters in each docking calculation obtained for a) caffeic acid, b) quercetin and c) sulphate.

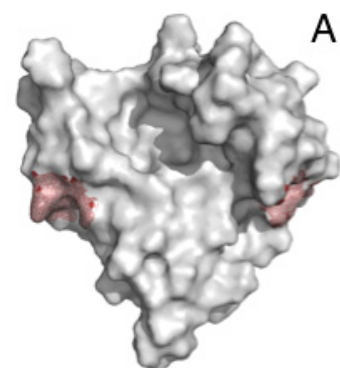

A

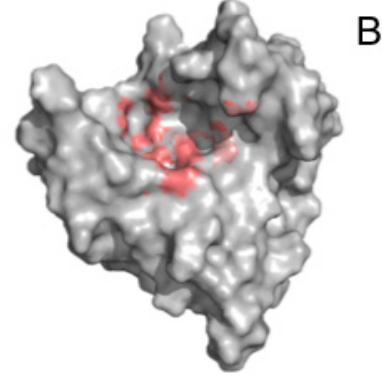

FIGURE 4
B

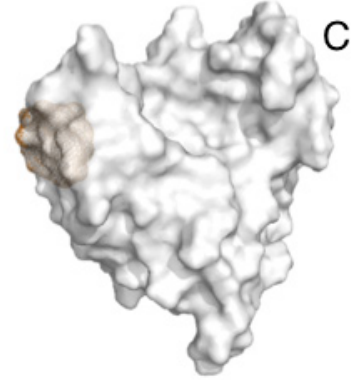

In red, the principal sites of interaction on the TLP (represented as surface in colour grey) with a) caffeic acid, b) quercetin and c) sulphate.

The fact that the complexes in which the ligand is positioned in the biggest cavity of the protein present the lowest energy of binding ( $75 \%$ of the models) seems to be favoured by the types of interactions that the ligand could develop on this site (see Fig. 5), a fact that will be analysed in the next section.

\section{DISCUSSION}

Despite the different nature of the ligands tested during the calculations, the interactions with the TLP measured at molecular level seem to clarify some aspects related to the haze phenomenon, which will be exposed in the following paragraphs in descendent order according to the average energy of binding of the complexes presented in Table 2.

In the sulphate molecule, the two major clusters are linked to the surface of the TLP without major steric hindrance, corresponding to the random coil formed from residue T136 to T141. The sulphate molecule, as has been reported, could be the potential X-factor responsible for triggering the haze phenomenon in wines (Pocock et al., 2007). In this sense, although the energy of binding predicted for this complex is higher in the present study, our results from working with the TLP structure only show that the interaction is less favoured in energetic terms and thereby that it is less probable that the complexes become stable over time.

Analysing the interactions described by the more populated clusters in the ligand caffeic acid, two zones of contact are favoured the most and are mainly defined by the carboxylate group of the molecule. The interactions are on the surface of the TLP, as has been shown in Fig. 4. The first cluster of 101 models orientated its carboxylate group to the positive residue $\mathrm{K} 172$, and the planar ring to the backbone of the protein between residues K159 and N165. The second, more populated cluster of 102 models, ranked as third in terms of the average energy of binding for this molecule $(-4.94 \mathrm{kcal} / \mathrm{mol})$. It orientated its carboxylate group to positive residue R142. The fact that the two most populated clusters are similar regarding their orientation is due to the nature of the molecule, which is essentially rigid with only two points of torsion and a stiff phenolic ring. This limits the number of interactions with the TLP, reflecting also on the small difference observed between the lowest and the average binding energies in the same family.

Although the identification of the specific residues involved in the recognition process between the TLP and the non-proteinaceous compounds has not been reported previously, the positive nature of these residues has been documented since 1996 (Siebert et al., 1996).

In the case of the ligand with the lowest energy of binding, the quercetin, the analysis for cluster 1 (Fig. 5b), with a binding average energy of $-7.31 \mathrm{Kcal} / \mathrm{mol}$, the lowest

TABLE 2

Lowest binding energy and average binding energy (kcal/ $\mathrm{mol}$ ) in the cluster of lowest energy of each ligand.

\begin{tabular}{lll}
\hline & $\begin{array}{l}\text { Lowest binding } \\
\text { energy (kcal/mol) }\end{array}$ & $\begin{array}{l}\text { Average binding } \\
\text { energy (kcal/mol) }\end{array}$ \\
\hline C family & $-5,17$ & $-5,15$ \\
Q family & $-7,38$ & $-7,31$ \\
S family & $-3,43$ & $-3,43$ \\
\hline
\end{tabular}


of the present study, shows that the chemical interactions are with residues of apolar nature inside the big cavity of the TLP. As already described by Siebert et al. (1996), these interactions of an apolar nature must be those that determine the binding of the ligands to the $\mathrm{P}$ residues on the protein (Siebert, 1999). In contrast, our results indicate that the relationship existing between the ligands and the protein is mainly with the residues Y98, Y200 and F118, and not with the $\mathrm{P}$ residues. It was observed that the $\mathrm{P}$ residues are not accessible for the quercetin in the big cavity of the TLP. However, it should be recognised that the interactions with the apolar side chains of those residues could be similar between Y, F and P. Mutational studies on the residues in the native systems (e.g. F119P) could corroborate the similarities or differences in energetic and structural terms.

In the second family (Fig. 5c), a kind of coupled interaction is observed between the ligand and the residues N179 and Y200, where the positive side chain of the N179 can interact with the negative net charge located in the extremities of the molecule. It is interesting to point out that the models belonging to cluster 5 (Figure 5e) describe a strong $\pi-\pi$ stacking interaction (at a very low distance of

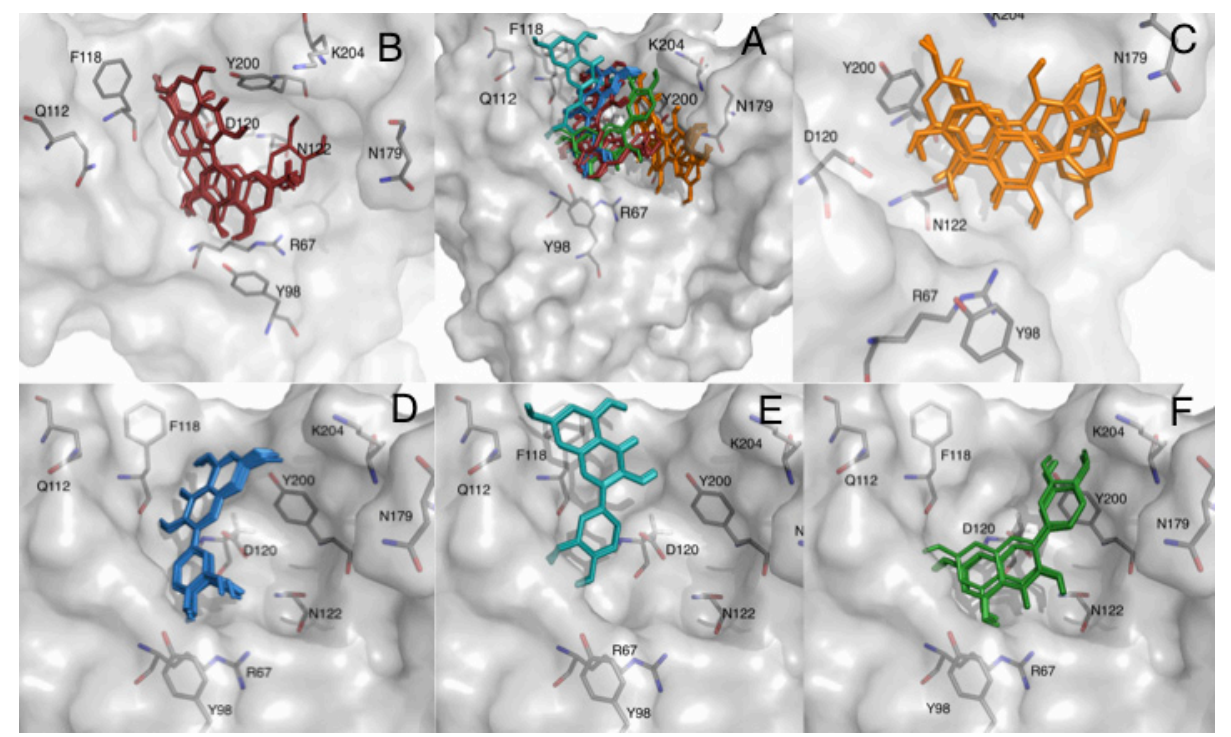

FIGURE 5

Modes of binding predicted for quercetin in the big cavity of the TLP. In a), the 287 models, in b), those structures that belong to cluster 1 , in c), those that belong to cluster 2 , in d), those that belong to cluster 3 , in e), those that belong to cluster 5 , and in f). those that belong to cluster 7 . For clarity, the hydrogen atoms of the protein are not shown.

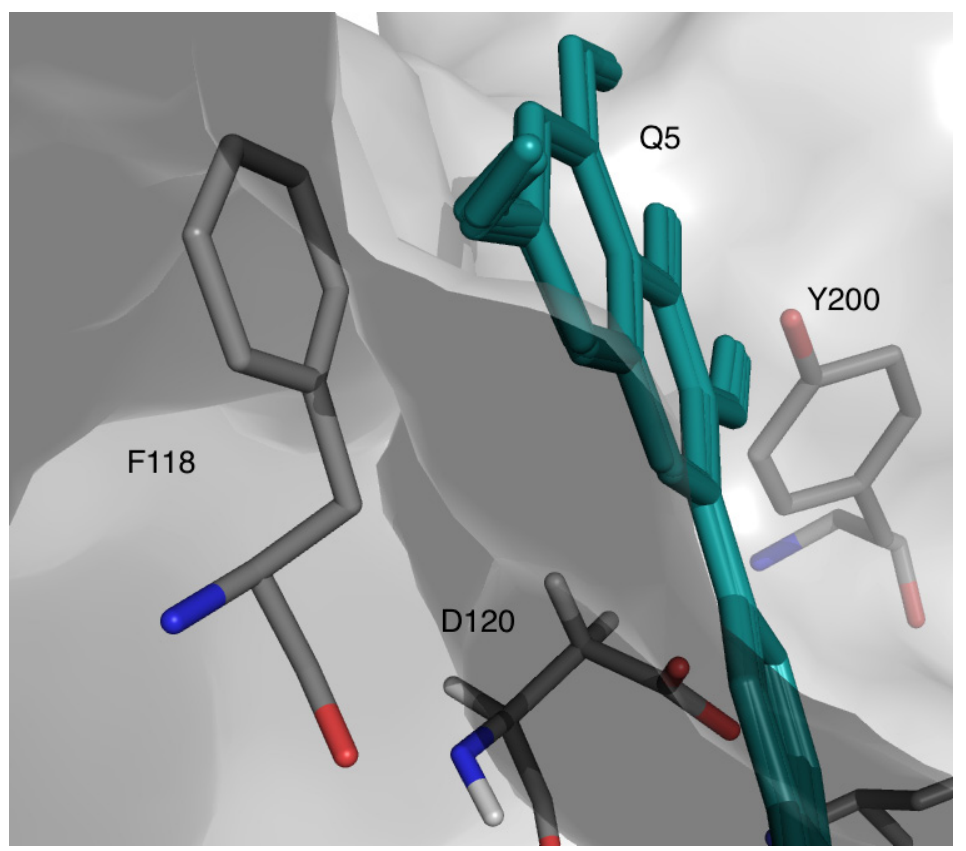

FIGURE 6

Close-up of the $\pi-\pi$ stacking interaction measured in the complexes between the ligand quercetin and residue F118 of the TLP. 
$3.6 \AA$ ) with the residue F118 (see Fig. 6). These kinds of interactions were described by Baxter et al. (1997) in an investigation of the relationship between polyphenols and the salivary proline-rich proteins.

Considering the above information, it seems to be important to highlight that the complexes that preferably are not linked to the bigger cavity of the TLP are less favoured in energetic terms than those that are able to reach it. And this fact could be the origin of the haze in the bottled wine, in which the time necessary to allow the appearance of these interactions between the molecules seems to be the crucial factor in making way for the formation of stable complexes between the TLP and their ligands. If this happens, it is expected that the complexes that could be formed between the globular or native protein and quercetin will be more stable and remains unaltered along the time. In this sense, a recent work postulates that the mechanism of haze formation seems to be independent of the protein (TLP or chitinase), and highlighted that only the TLP isoforms that denature will participate in haze formation (Van Sluyter et al., 2015). Based on the energetic results and from the previous experimental characterisation of TLP, which defined this structure as stable, we postulate that the mechanism of interaction observed between the TLP and quercetin could be responsible for modulating the formation of the haze in the long term, mediated by the presence of TLP. However, it cannot be discarded that a sensitive fraction of the TLP to winemaking could be denatured, and that in that unfolded state its reactive behaviour is similar to that of the chitinases.

The results presented here could explain the experimental observations relating to the formation of haze in clear beverages, with other phenolic compounds interacting through hydrophobic bonding, a theme reviewed extensively by Siebert (1999). This finding relates to the hypothesis of Marangon et al. (2011), namely the that TLP could precipitate in the long term with a slower and possibly different mechanism of chitinases. The unequal behaviour between these molecules has also been documented by Falconer et al. (2010) in relation to the thermal stability of the TLP and chitinase measured by its half-life. From this work (Falconer et al., 2010), the prediction of the half-life of chitinases in the model wine at $30^{\circ} \mathrm{C}$ was 4.7 days, and only $14 \mathrm{~h}$ at $35^{\circ} \mathrm{C}$, whereas the predicted half-life of TLP was 45 years at $35^{\circ} \mathrm{C}$. In view of this, the role that TLP plays in haze formation is unclear.

On the basis of these results it is proposed that there is a differentiated mechanism of haze formation between the TLP and chitinases, which depends on the ability of the ligand molecules to reach the big cavity of the TLP until get stable complexes in energetic terms. It is suggested that, in this mechanism, the sulphate, and in general any compound that cannot be allocated in contact with the residues present in the big cavity of the TLP, would have a secondary role in the formation of the turbidity observed in the bottled product.

\section{CONCLUSIONS}

Based on the theoretical results presented in this work, it is proposed that the TLP is able to form complexes with those phenolic compounds that reach the hydrophobic molecular cavity and interact in different ways with the residues Y98,
Q112, F118, D120, N122, N179, Y200 and K204, according to their nature. This area of difficult access in steric terms represents the most favoured interaction region in terms of energy, and probably the most stable are for the stabilisation of the complexes in dynamic terms in the long term.

In this study only the quercetin was capable of reaching the hydrophobic big cavity on the TLP, presenting the lowest energy of binding $(-7.38 \mathrm{Kcal} / \mathrm{mol})$. The caffeic acid and the sulphate molecule could interact in the peripheral zones of the TLP, forming complexes with higher energies of binding than those observed in the complexes with quercetin as ligand. This supports the fact that, in the long term, the TLP in complex with phenolic compounds could be responsible for the haze observed in the bottled wine. These results do not contradict the experimental evidence affirming that the start of haze is triggered by the presence of chitinases or by the unfolded TLP, an aspect that is not discussed in this work.

From this study it was possible to establish the TLP residues that could interact with the phenolic ligand to establish interactions of the type $\pi-\pi$ stacking in the big cavity, namely Y98, F118 and Y200. This kind of interaction was described as necessary in these complexes by Baxter et al. (1997), but not characterised in detail until now.

It is hoped that the detailed results in this work obtained at the molecular level can be useful in moving forward to the definition of the mechanism that governs the phenomenon of turbidity in bottled white wine, thereby contributing to the development of new techniques and technologies to replace fining treatments.

\section{LITERATURE CITED}

Ahlrichs, R., Bär, M., Häser, M., Horn, H. \& Kölmel, C., 1989. Electronic structure calculations on workstation computers: The program system turbomole. Chem. Phys. Lett. 162(3), 165-169.

Baxter, N.J., Lilley, T.H., Haslam, E. \& Williamson, M.P., 1997. Multiple interactions between polyphenols and a salivary proline-rich protein repeat result in complexation and precipitation. Biochem. 36(18), 5566-5577.

Bernstein, F.C., Koetzle, T.F., Williams, G.J., Meyer, E.F., Brice, M.D., Rodgers, J.R., Kennard, O., et al., 1977. The Protein Data Bank: A computer-based archival file for macromolecular structures. J. Mol. Biol. 112(3), 535-542.

Besse, C., Clark, A. \& Scollary, G., 2000. Investigation of the role of total and free copper in protein in haze formation. Aust. Grapegrow. Winemak. 437, 19-20.

Brooks, B.R., Bruccoleri, R.E., Olafson, B.D., States, D.J., Swaminathan, S. \& Karplus, M., 1983. CHARMM: A program for macromolecular energy, minimization, and dynamics calculations. J. Comput. Chem. 4(2), 187-217.

Dawes, H., Boyes, S., Keene, J. \& Heatherbell, D., 1994. Protein instability of wines: Influence of protein isolelectric point. Am. J. Enol. Vitic. 45(3), 319-326.

Distinto, S., Meleddu, R., Yanez, M., Cirilli, R., Bianco, G., Sanna, M.L., Arridu, A., Cossup, P., Cottiglia, F., Faqqi, C., Ortuso, F., Alcaro, S., Maccioni, E., 2015. Drug design, synthesis, in vitro and in silico evaluation of selective monoaminoxidase B inhibitors based on 3-acetyl-2dichlorophenyl-5-aryl-2,3-dihydro-1,3,4-oxadiazole chemical scaffold. Eur. J. Med. Chem. 108, 542-552.

Dolinsky, T.J., Nielsen, J.E., McCammon, J.A. \& Baker, N.A., 2004. PDB2PQR: An automated pipeline for the setup of Poisson-Boltzmann electrostatics calculations. Nucleic Acids Res. 32(Web Server issue), W665-W667. 
Dufrechou, M., Vernhet, A., Roblin, P., Sauvage, F.X. \& Poncet-Legrand, C., 2013. White wine proteins: How does the $\mathrm{pH}$ affect their conformation at room temperature? Langmuir 29(33), 10475-10482.

Falconer, R.J., Marangon, M., Van Sluyter, S.C., Neilson, K.A., Chan, C. \& Waters, E.J., 2010. Thermal stability of thaumatin-like protein, chitinase, and invertase isolated from Sauvignon blanc and Semillon juice and their role in haze formation in wine. J. Agric. Food Chem. 58(2), 975-980.

Heatherbell, D.A., 1976. Haze and sediment formation in clarified apple juice and apple wine. Alimenta 15, 151-154

Hellberg, S., Sjoestroem, M., Skagerberg, B. \& Wold, S., 1987. Peptide quantitative structure-activity relationships, a multivariate approach. J. Med. Chem. 30(7), 1126-1135.

Høj, P.B., Tattersall, D.B., Adams, K., Pocock, K.F., Hayasaka, Y., Van Heeswijck, R. \& Waters, E.J., 2000. The 'haze proteins' of wine - a summary of properties, factors affecting their accumulation in grapes, and the amount of bentonite required for their removal from wine. In Proc. ASEV 50th Anniv. Meeting, 2000, Seattle, Washington, USA. pp. 149 - 154

Höltje, H.-D., Sippl, W., Rognan, D. \& Folkers, G., 2008. Molecular modeling: Basic principles and applications. John Wiley \& Sons. Available at: https://books.google.com/books?id=xkzquS1LRGIC\&pgis=1 (accessed 19 January 2016)

Huey, R., Morris, G.M., Olson, A.J. \& Goodsell, D.S., 2007. A semiempirical free energy force field with charge-based desolvation. J. Comput. Chem. 28(6), 1145-1152.

Jung, Y., Lochan, R., Dutoi, A. \& Head-Gordon, M., 2004. Scaled oppositespin second Moller-Plesset correlation energy: An economical electronic structure method. J. Chem. Phys. 121(20), 9793.

Kendall, R.A., Dunning, T.H. \& Harrison, R.J., 1992. Electron affinities of the first-row atoms revisited. Systematic basis sets and wave functions. J. Chem. Phys. 96(9), 6796.

Kristensen, A.S., Hansen, K.B., Naur, P., Olsen, L., Kurtkaya, N.L., Dravid, S.M., Kvist, T., et al., 2015. Pharmacology and structural analysis of ligand binding to the orthosteric site of glutamate-like GluD2 receptors. Mol. Pharmacol. 89(2), 253-262.

Li, H., Robertson, A.D. \& Jensen, J.H., 2005. Very fast empirical prediction and rationalization of protein $\mathrm{pK}$ a values. Proteins 61(4), 704-721.

Lin, J.H., 2016. Review structure- and dynamics-based computational design of anticancer drugs. Biopolymers 105(1), 2-9.

Marangon, M., Sauvage, F.X., Waters, E.J. \& Vernhet, A., 2011. Effects of ionic strength and sulfate upon thermal aggregation of grape chitinases and thaumatin-like proteins in a model system. J. Agric. Food Chem. 59(6), $2652-2662$

Marangon, M., Van Sluyter, S.C., Neilson, K.A., Chan, C., Haynes, P.A., Waters, E.J. \& Falconer, R.J., 2011. Roles of grape thaumatin-like protein and chitinase in white wine haze formation. J. Agric. Food Chem. 59(2), 733-740.

Marangon, M., Van Sluyter, S.C., Waters, E.J. and Menz, R.I. (2014), "Structure of haze forming proteins in white wines: Vitis vinifera thaumatinlike proteins.”, PloS One, Vol. 9 No. 12, p. e113757.

Matta, C.F. \& Boyd, R.J., 2007. The quantum theory of atoms in molecules: From solid state to DNA and drug design. Available at: https://books. google.com/books?hl=es\&lr=\&id=7mE9rwz6P04C\&pgis=1 (accessed 24 July 2015)

Morris, G.M., Goodsell, D.S., Halliday, R.S., Huey, R., Hart, W.E., Belew, R.K. \& Olson, A.J., 1998. Automated docking using a Lamarckian genetic algorithm and an empirical binding free energy function. J. Comput. Chem. 19(14), 1639-1662.
Pellerin, P., Waters, E., Brillouet, J.M. \& Moutounet, M., 1994. Effet de polysaccharides sur la formation de trouble protéique dans un vin blanc. J. Int. Sci. Vigne Vin (28)3, 213-225.

Peng, Z., Pocock, K.F., Waters, E.J., Francis, I.L. \& Williams, P.J., 1997. Taste properties of grape (Vitis vinifera) pathogenesis-related proteins isolated from wine. J. Agric. Food Chem. 45(12), 4639-4643.

Pettersen, E.F., Goddard, T.D., Huang, C.C., Couch, G.S., Greenblatt, D.M., Meng, E.C. \& Ferrin, T.E., 2004. UCSF Chimera - A visualization system for exploratory research and analysis. J. Comput. Chem. 25(13), 1605-1612.

Picinelli, A., Bakker, J. \& Bridle, P., 1994. Model wine solutions: Effect of sulphur dioxide on colour and composition during ageing. Vitis 33, 31-35.

Pocock, K.F., Alexander, G.M., Hayasaka, Y., Jones, P.R. \& Waters, E.J., 2007. Sulfate - A candidate for the missing essential factor that is required for the formation of protein haze in white wine. J. Agric. Food Chem. 55(5), 1799-1807.

Reed, A.E., Weinstock, R.B. \& Weinhold, F., 1985. Natural population analysis. J. Chem. Phys. 83, 735-746.

Siebert, K.J., 1999. Effects of protein-polyphenol interactions on beverage haze, stabilization, and analysis. J. Agric. Food Chem. 47(2), 353-362.

Siebert, K.J., Troukhanova, N.V. \& Lynn, P.Y., 1996. Nature of polyphenolprotein interactions. J. Agric. Food Chem. 44(1), 80-85.

Tattersall, D.B., Van Heeswijck, R. \& Høj, P.B., 1997. Identification and characterization of a fruit-specific, thaumatin-like protein that accumulates at very high levels in conjunction with the onset of sugar accumulation and berry softening in grapes. Plant Physiol. 114(3), 759-769.

Tattersall, D.B., Pocock, K.F., Hayasaka, Y., Adams, K., Van Heeswijck, R., Waters, E.J. \& Høj, P.B., 2001a. Pathogenesis related proteins - Their accumulation in grapes during berry growth and their involvement in white wine heat instability. Current knowledge and future perspectives in relation to winemaking practices. Mol. Biol. Biotechnol. Grapevine 183-201.

Van Sluyter, S.C., McRae, J.M., Falconer, R.J., Smith, P.A., Bacic, A., Waters, E.J. \& Marangon, M., 2015. Wine protein haze: Mechanisms of formation and advances in prevention. J. Agric. Food Chem. 63(16), 4020 4030 .

Wang, T., Wu, M.-B., Zhang, R.-H., Chen, Z.-J., Hua, C., Lin, J.-P. \& Yang, L.-R., 2016. Advances in computational structure-based drug design and application in drug discovery. Curr. Top. Med. Chem. 16(9), 901-916.

Waters, E.J., Alexander, G., Muhlack, R., Pocock, K., Colby, C., O’Neil, B. \& Høj, P., 2005. Preventing protein haze in bottled white wine. Proteins 11(2), 215-225

Waters, E.J., Peng, Z., Pocock, K. \& Williams, P., 1995. Proteins in white wine, I: Procyanidin occurrence in soluble proteins and insoluble protein hazes and its relationship to protein instability. Aust. J. Grape Wine Res. $1(2), 86-93$

Wishart, D.S., Knox, C., Guo, A.C., Shrivastava, S., Hassanali, M., Stothard, P., Chang, Z., et al., 2006. DrugBank: A comprehensive resource for in silico drug discovery and exploration. Nucleic Acids Res. 34(Database issue), D668-D672

Xiao, Z., Morris-Natschke, S.L. \& Lee, K.-H., 2016. Strategies for the optimization of natural leads to anticancer drugs or drug candidates. Med. Res. Rev. 36(1), 32-91.

Yokotsuka, K., Ebihara, T. \& Sato, T., 1991. Comparison of soluble proteins in juice and wine from Koshu grapes. J. Ferment. Bioeng. 71(4), 248-253.

Yuan, Y., Pei, J. \& Lai, L., 2013. Binding site detection and druggability prediction of protein targets for structure-based drug design. Curr. Pharm. Des. 19(12), 2326-2333. 